\title{
Analysis on Privacy and Reliability of Ad Hoc Network-Based in Protecting Agricultural Data
}

\author{
Xiangju Liu* \\ Guizhou University of Finance and Economics, Guizhou 550004, China
}

\begin{abstract}
To analyze the privacy and reliability of Ad Hoc Network-based algorithms used in agricultural wireless data transmission, the paper introduces the routing protocols including DSDV, AODV and DSR, based on which a farmland model is built to simulate the speed and density of mobile node, and regards the success rate of data transmission (R/S Rate), average latency of end-to-end (E2E Delay) and average hops as the evaluation indicator of farmland information transfer system. As a result of the analysis, the privacy and reliability are influenced by the speed and density of mobile nodes; the higher the speed is and the lower the density is, the lower the R/S Rate is; AODV routing protocol is better in $\mathrm{R} / \mathrm{S}$ Rate while DSDV routing protocol does better in both E2E Delay and average hops. It is concluded that DSDV routing protocol suits most the Ad Hoc network-based farmland information transfer system.
\end{abstract}

Keywords: Ad Hoc Network, DSDV routing protocol, AODV routing protocol, DSR routing protocol.

\section{INTRODUCTION}

With the rapid development of the Internet technology in recent years, the Wi-Fi technology receives an equally fast development and has increased its share in the Internet market. Compared with wired network, the mobile network has its own characteristics; its topological structure would change with the movement of mobile nodes, which is impossible for the wired network, while it can provide services for its clients fast, flexibly and conveniently, which makes it the future of the Internet. Ad Hoc is a special type of wireless communication mode, which is multi-hop temporary autonomous system; it is a spawning network of the ALOHA built in the United States in 1968 which is a single-hot network connecting each node so as to communicate, and the PR (Packet Radio) built in the United States in 1973 which does not need to communicate based on each connecting node $\lceil 1\rceil$. In the Ad Hoc network, each node is equal, which means that Ad Hoc does not need to build extra node and can resist being damaged [2].

In 2002, Inter built a wireless vineyard in Oregon, which was the first time in human history to employ the Internet on agriculture and started the process of agriculture informatization. From then on, Wi-Fi technology is gradually used on agriculture; take the most striking examples, checking the room temperature, water-saving irrigation, environmental monitoring and physiological and ecological monitoring of plants and animals. Precision agriculture is the future of global farming; also, it is the key to accelerate to adjust traditional agriculture and improve overall agricultural production capacity in our country [3]. Wi-Fi technology, as the key to precision agriculture, is quite promising; Ad Hoc network, as a part of Wi-Fi technology, can be potential enough for the farmland information transfer. This paper is to analyze the privacy and reliability of algorithm on Ad Hoc Network-based agricultural data [4].

\section{SEVERAL ROUTING PROTOCOLS IN AD HOC NETWORK}

After years' development, many routing protocols of Ad Hoc Network have been set up; based on different discovery strategies, the routing protocols of Ad Hoc Network can be divided into table-driven and on-demand routing protocols; based on different topological structures, the routing protocols of Ad Hoc network can be divided into hierarchical and flat routing protocols; based on whether GPS is used, the routing protocols of Ad Hoc Network can be divided into location and non-location aided routing protocols [5]. The detailed classification is shown is Fig. (1).

In this paper, the author focuses on the most commonly used routing protocols in Ad Hoc network-DSDV routing protocol, which is a table-driven routing protocol; AODV routing protocol and DSR routing protocol, which are two on-demand routing protocols.

\subsection{DSDV Routing Protocol}

DSDV (Destination Sequenced Distance Vector) is a classic table-driven routing protocol coming from RIP; each of its nodes can make a routing table like the one in Table 1. DSDV routing protocol is based on the tradition BellmanFord and different from traditional WRP routing protocol. In the DSDV table, data including destination node, next step node, step length and destination node ID are included. Se- 


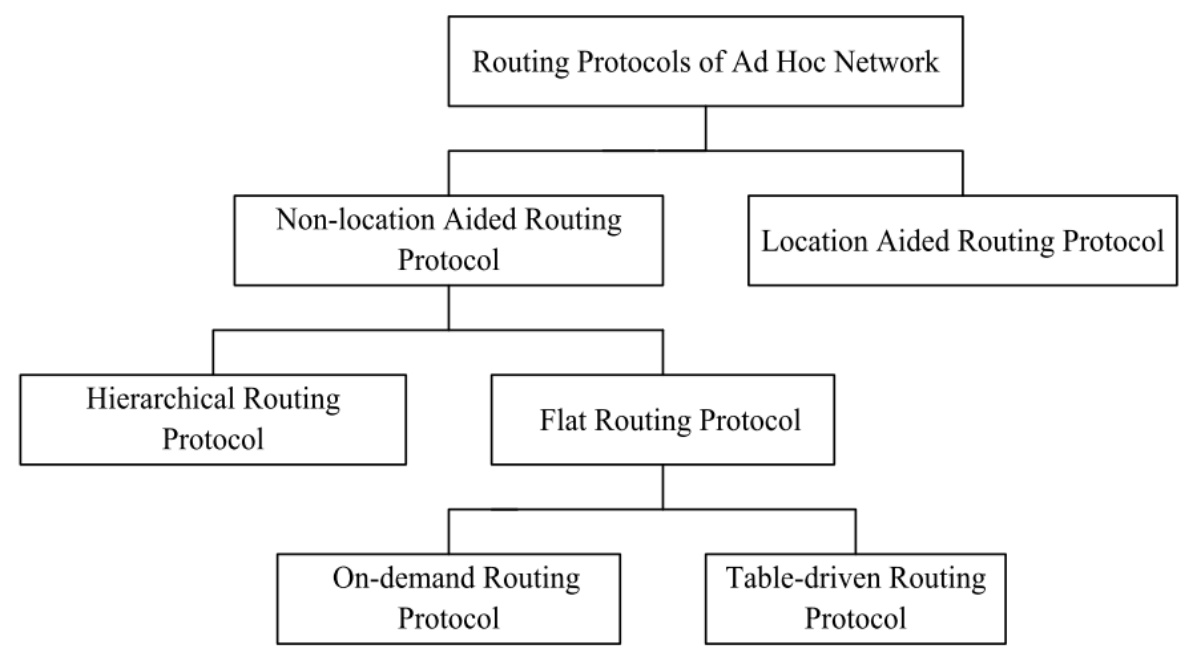

Fig. (1). The classification of network routing protocol of Ad HOC.

ries number for DSDV is used to distinguish the old and new routing protocol to avoid routing loop. DSDV table updates in two ways: the topological structure update the whole table when web changes at a high speed; or the topological structure update part of the table when web is fairly stable. The algorithm of DSDV goes as follows. When a component which does not find its routing protocol arrives at a certain nodes, the component would stay while the node gives an order to find its routing protocol; until the other routing protocol response (when there are more components than the node can store, new component would not be let in), the component would be put right the place the routing protocol orders and then give the end to the destination node.

Table 1. Routing table of DSDV.

\begin{tabular}{|c|c|c|c|c|c|}
\hline Destination & Next & Step & Routing & Creation & Destination \\
\hline \multirow{2}{*}{ Node } & Hop & & & & \\
\cline { 2 - 6 } & Node & Length & Loop & Time & Node ID \\
\hline A & A & 0 & A-332 & 001000 & Ptr-A \\
\hline B & B & 1 & B-168 & 001200 & Ptr-B \\
\hline C & B & 3 & C-489 & 001200 & Ptr-C \\
\hline D & B & 4 & D-265 & 001200 & Ptr-C \\
\hline
\end{tabular}

The advantages of DSDV routing protocol lies in its easy operation; it is often used in simple and small network; it is not suitable for the fast changing network; it does not support one-way route [6].

\subsection{AODV Routing Protocol}

AODV(Ad Hoc On-demand Distance Vector) is an ondemand routing protocol of the Ad Hoc network, which means that it only need to protect the information about the route of the routing protocol. That is to say, when it sends packets to destination nodes, the original node has to find the route by searching in the Internet. Conversely, many routing protocols of the Internet is prior, which means that they do not need to rely on whether they need to send packets or not but on the routing table including all the information about

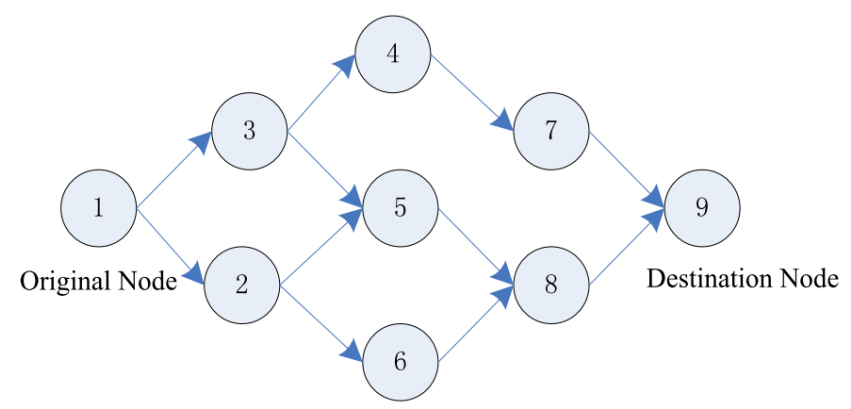

Fig (2). Requests from AODV Routing Protocol.

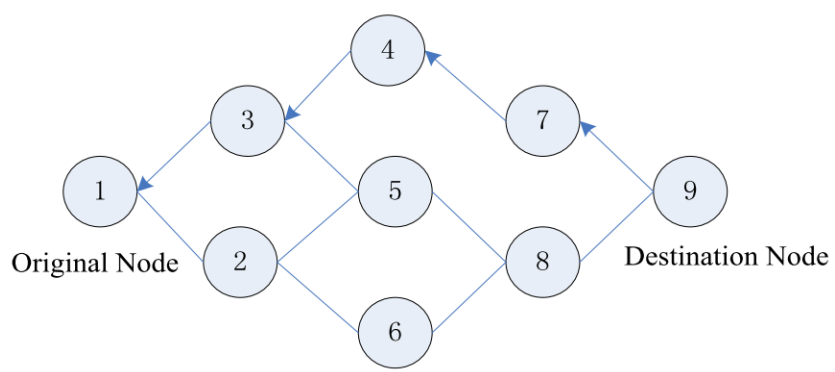

Fig. (3). Transferences of answers in AODV routing protocol.

nodes to nodes. Each two of the nodes continue to exchange information in a certain period of time so as to update the routing table and reflect the topological structure on the table. In this way, the information on the routing table can always be consistent, timely and correct [7]. Just as the name of AODV routing protocol indicates, it is a flat distance vector. The request from routing protocol and the answer to it are demonstrated in Figs. (2) and (3). 

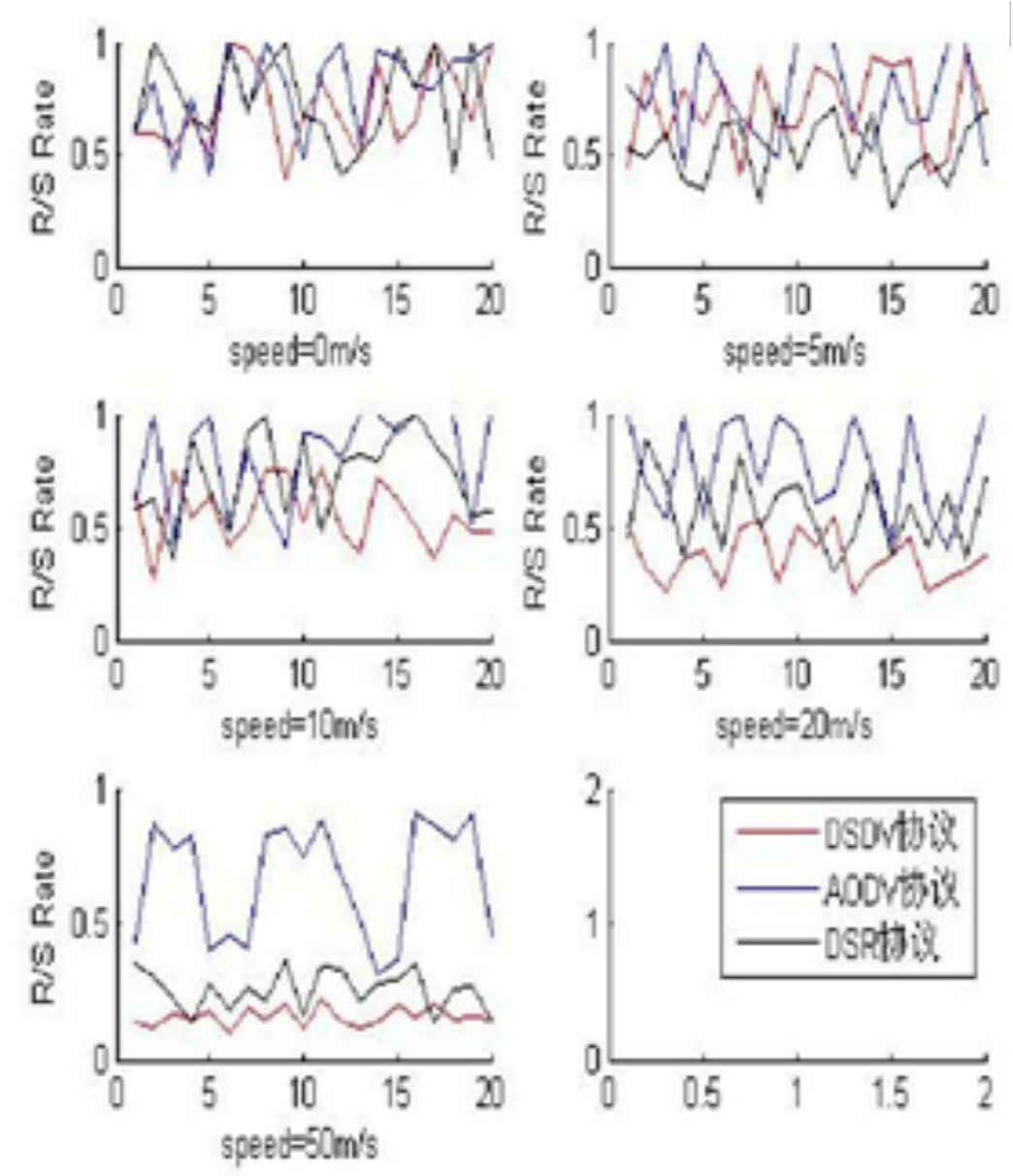

Fig. (4). Results of a simulation experiment.

\subsection{DSR Routing Protocol}

DSR (Dynamic Source Routing), or dynamic source routing protocol, is a simple and efficient routing protocol specially designed for multi-hop wireless Ad Hoc networks. DSR routing protocol has two main mechanisms - route discovery mechanism and route maintenance mechanism. Route discovery mechanism is used when the original node is to send a packet to the destination node but don't know how to arrive. When the original node is being sent to the destination node, the route maintenance mechanism would detect those failed routes due to changes in topological structure, send to destination node the information of packet and readjust the routing protocol to find out a new route [8]. In the DSR routing protocol, route discovery and maintenance mechanisms operate completely on demand rather than according to some kind of classification, such as, broadcasting groups or linkage detection groups. All states of the DSR routing protocol is "soft", which means that the loss of any state will not influence the correct its operation for all states are operated on demand and any state on demand can be restored easily in a short time as long as needed. The route discovery and maintenance mechanisms help DSR routing protocol make the one-way and asymmetry routing protocols more easily to operate.

\section{SIMULATION EXPERIMENTS ON THE ROUTING PROTOCOLS AND THE RESULTS}

There are now many excellent simulation software including the widely used OPNET, NS-2, MATLAB and SPW. In this paper, the author employs NS-2, whose detailed information about its usage and widget for routing protocols are not important and are not to be illustrated, to carry simulation experiments.

The author places randomly 100 mobile nodes in a experimental farmland radiating $1 \mathrm{~km}$. The experiment results are going to use the following evaluation radiators including $\mathrm{R} / \mathrm{S}$ rate, E2E delay and average hops.

\subsection{Simulation Experiments on Speed of Mobile Node}

The author set the speed of mobile node as $0 \mathrm{~m} / \mathrm{s}, 5 \mathrm{~m} / \mathrm{s}$, $10 \mathrm{~m} / \mathrm{s}, 20 \mathrm{~m} / \mathrm{s}$ and $50 \mathrm{~m} / \mathrm{s}$ and gets results demonstrated in Fig. (4). The other results of simulation experiments are also demonstrated in figures similar to Fig. (4), which the author is not to provide due to word limit of the paper but to make a conclusion in Table 2.

From Table 2, it is clearly shown, that, as for $\mathrm{R} / \mathrm{S}$ rate, AODV routing protocol $(76.24 \%)$ is better than the other 
Table 2. Results of simulation experiments.

\begin{tabular}{|c|c|c|c|c|c|c|}
\hline Speed & $\mathbf{R} / \mathbf{S}$ & $0 \mathrm{~m} / \mathrm{s}$ & $5 \mathrm{~m} / \mathrm{s}$ & $10 \mathrm{~m} / \mathrm{s}$ & $20 \mathrm{~m} / \mathrm{s}$ & $50 \mathrm{~m} / \mathrm{s}$ \\
\hline \multirow{3}{*}{ DSDV } & $\mathrm{R} / \mathrm{S}$ Rate & $71.1 \%$ & $66.2 \%$ & $52.3 \%$ & $38.1 \%$ & $17.5 \%$ \\
\hline & E2E Delay & 0.035 & 0.045 & 0.057 & 0.160 & 0.285 \\
\hline & Average Hops & 3.5 & 3.1 & 2.5 & 3.3 & 3.8 \\
\hline \multirow{3}{*}{ AODV } & $\mathrm{R} / \mathrm{S}$ Rate & $76.3 \%$ & $84.3 \%$ & $81.0 \%$ & $78.1 \%$ & $61.2 \%$ \\
\hline & E2E Delay & 0.249 & 0.134 & 0.261 & 0.224 & 0.209 \\
\hline & Average Hops & 6.7 & 5.4 & 4.4 & 3.8 & 5.9 \\
\hline \multirow{3}{*}{ DSR } & $\mathrm{R} / \mathrm{S}$ Rate & $80.1 \%$ & $49.2 \%$ & $70.0 \%$ & $59.6 \%$ & $25.1 \%$ \\
\hline & E2E Delay & 1.241 & 1.345 & 1.562 & 3.701 & 6.521 \\
\hline & Average Hops & 17.6 & 36.1 & 37.2 & 35.3 & 89.7 \\
\hline
\end{tabular}

Table 3. Results of simulation experiments on density of mobile node.

\begin{tabular}{|c|c|c|c|c|c|c|c|}
\hline Speed & $\mathbf{R} / \mathbf{S}$ & $10 \mathrm{~m}$ & $20 \mathrm{~m}$ & $40 \mathrm{~m}$ & $60 \mathrm{~m}$ & $80 \mathrm{~m}$ & $100 \mathrm{~m}$ \\
\hline \multirow{3}{*}{ DSDV } & R/S Rate & $84.1 \%$ & $73.2 \%$ & $75.4 \%$ & $74.4 \%$ & $73.7 \%$ & $70.3 \%$ \\
\hline & E2E Delay & 0.035 & 0.038 & 0.034 & 0.039 & 0.043 & 0.034 \\
\hline & Average Hops & 3.2 & 3.4 & 3.8 & 4.0 & 4.3 & 4.5 \\
\hline \multirow{3}{*}{ AODV } & $\mathrm{R} / \mathrm{S}$ Rate & $86.7 \%$ & $83.2 \%$ & $79.2 \%$ & $75.9 \%$ & $75.8 \%$ & $72.9 \%$ \\
\hline & E2E Delay & 0.214 & 0.242 & 0.287 & 0.259 & 0.357 & 0.401 \\
\hline & Average Hops & 4.5 & 4.6 & 4.7 & 5.6 & 5.2 & 5.6 \\
\hline \multirow{3}{*}{ DSR } & R/S Rate & $81.7 \%$ & $80.6 \%$ & $77.2 \%$ & $70.9 \%$ & $72.1 \%$ & $65.9 \%$ \\
\hline & E2E Delay & 1.210 & 1.364 & 1.874 & 2.014 & 2.471 & 3.062 \\
\hline & Average Hops & 17.3 & 18.2 & 19.6 & 21.6 & 25.4 & 30.1 \\
\hline
\end{tabular}

two-routing protocols DSDV (49.05\%) and DSR (56.84\%); that the results of simulation experiments all indicate a trend for three routing protocols - the higher the speed of node is, the lower the $\mathrm{R} / \mathrm{S}$ rate is; or the privacy and reliability of three routing protocols are influenced by speed of node, that is to say, the higher the speed is, the worse the privacy and reliability are. What's more, Table 2 shows that, as for E2E delay and average hops, DSDV and AODV routing protocols are better while the former much better than the latter; that DSR routing protocol is the worst of three. All these results are directly influenced by the algorithm of each routing protocol. In general, AODV routing protocol is the most stable one; the DSDV routing protocol has advantages over the other two on both E2E delay and average hops, but has disadvantages on $\mathrm{R} / \mathrm{S}$ rate compared with AODV routing protocol: therefore, AODV routing protocol, from a general perspective, is better than the other two routing protocolsDSDV and DSR.

\subsection{Simulation Experiments on Density of Mobile Node}

The author chooses 20 in 100 nodes and carries the simulation experiments on density of mobile node by broadening the distance between each two of them for $10 \mathrm{~m}, 20 \mathrm{~m}, 40 \mathrm{~m}$,
$60 \mathrm{~m}, 80 \mathrm{~m}$ and $100 \mathrm{~m}$. The results of the experiments are demonstrated in Table 3.

From Table 3, it can be known, that as for the R/S Rate, AODV routing protocol $(78.95 \%)$ is better than the other two - routing protocols DSDV (75.18\%) and DSR (74.73\%); and that the R/S Rate goes down along with the lowering of the node density is; and that DSDV routing protocol change least in this process. What's more, it is demonstrated that, as for the E2E Delay, DSDV routing protocol $(0.037 \mathrm{~s})$ is better than the other two-routing protocols AODV (0.293 s) and DSR (1.999 s); as for the average hops, DSDV routing protocol is better than routing protocols AODV and DSR.

\section{DISCUSSION AND CONCLUSION}

The paper carries simulation experiments and calculation on the speed and density of mobile node of farmland in real life; according to the result, the privacy and reliability are influenced by the speed and density of mobile nodes; that the higher the speed is and the lower the density is, the lower the $\mathrm{R} / \mathrm{S}$ Rate is; as for the R/S Rate, AODV routing protocol is better than the other two-routing protocols DSDV and DSR; as for both the E2E Delay and the average hops, DSDV routing protocol is better than AODV routing protocol followed 
by DSR routing protocol; in the farmland information transfer system, farmland environment will not change along with the time; almost all mobile nodes move at very little speed while some even stay still; also, the density of mobile nodes is quite low. Therefore, DSDV routing protocol suits most the Ad Hoc network-based farmland information transfer system while, if taking the privacy and reliability of farmland information, i.e. the R/S Rate, AODV routing protocol is the best choice.

\section{CONFLICT OF INTEREST}

The author confirms that this article content has no conflict of interest.

\section{ACKNOWLEDGEMENTS}

This work is supported by the Project of Science \& Technology Research and Development in Zhang Jiakou City, No. 1311020C; the Rural Medical \& Health Knowledge Base and the Remote Medical Service System Together with Its Application----The Project of Ministry of Science and Technology, No. 2012BAH05F04; the Project of Science \& Technology Research and Development in Zhang Jiakou City, No. 1311033B The Major Projects in Hebei North University, No.1311033B; The Major Scientific
Research Projects in Higher School in Hebei Province, No. ZD20131085.

\section{REFERENCES}

[1] P. Liu, Design and Operation of Wi-Fi-based Video Monitoring System. Hangzhou: Zhejiang University, 2006

[2] X. M. Fan, Z. G. Shan, and B. X. Zhang, "State-of-the-Art of the Architecture and Techniques for Delay-Tolerant Networks", Chinese Journal of Electronics, vol. 36, no. 1, pp. 161-170, 2008

[3] K. Zheng and N. Wang, "New AODV based clustering routing strategy", Journal of Communications, vol. 27, no. 1, pp. 132-139, 2006

[4] Z. L. Liu, "The Dynamic Analysis of China's Energy-EconomyEnvironment System: VAR and VEC Modeling", Advances in Information Sciences and Service Sciences, vol. 4, no. 14, pp. 210218, 2012. doi: 10.4156/AISS.vol4.issue 14.25

[5] Z. J. Xu, Research and Operation on Wifi-based Remote Control Platform. Nanjing: Nanjing University of Aeronautics and Astronatutics, 2005.

[6] J. L. Wang and C. G. Wang, Ad Hoc Mobile Wireless Networks. Beijing: National Defense Industry Press, 2004.

[7] T. L. Zhang, Z. C. Li, and M. Liu, "Partial Connection Availability Modeling for Wireless Networks", Chinese Journal of Computers, vol. 30, no. 4, pp. 505-513, 2007

[8] J. Su, A. Chin, and A. Popivanova, "User Mobility for Opportunistic Ad-Hoc Networking", in Proceedings of the 6th IEEE Workshop on Mobile Computing System and Applications (WMCSA), UK, December 2004, pp. 41-50. 